\title{
Linguistic Interpretation of Mathematical Morphology
}

\author{
Agustina Bouchet $^{1,2}$, Gustavo Meschino ${ }^{3}$, Marcel Brun ${ }^{1}$, Rafael Espin Andrade ${ }^{4}$, Virginia Ballarin ${ }^{1}$ \\ ${ }^{1}$ Universidad Nacional de Mar de Plata, Mar de Plata, Argentina. \\ ${ }^{2}$ CONICET, Mar de Plata, Argentina. \\ ${ }^{3}$ Universidad Nacional de Mar de Plata, Mar de Plata, Argentina. \\ ${ }^{4}$ ISPJAE, Universidad Técnica de La Habana, Cuba. \\ abouchet@fi.mdp.edu.ar, gmeschin@fi.mdp.edu.ar, mbrun@fi.mdp.edu.ar, \\ espin@ind.cujae.edu.cu, vballari@fi.mdp.edu.ar
}

\begin{abstract}
Mathematical Morphology is a theory based on geometry, algebra, topology and set theory, with strong application to digital image processing. This theory is characterized by two basic operators: dilation and erosion. In this work we redefine these operators based on compensatory fuzzy logic using a linguistic definition, compatible with previous definitions of Fuzzy Mathematical Morphology. A comparison to previous definitions is presented, assessing robustness against noise.
\end{abstract}

Keywords: Fuzzy Logic, Compensatory Fuzzy Logic, Mathematical Morphology, Fuzzy Mathematical Morphology.

\section{Introduction}

Mathematical Morphology (MM) is a theory based on concepts of geometry, algebra, topology and the theory of sets, created to characterize physical and structural properties of various materials [1-3]. MM allows processing images with different objectives, such as enhancing diffuse zones, segmenting objects, detecting edges, analyze structures, among others. The central idea of the MM is to examine the geometric structures of an image by superposing with small localized patterns, called structuring elements (SE), in different parts of it [1]. The two basic operations of the MM are dilation and erosion. Its composition generates filtering operators, for example, opening and closing.

Another existing approach in the literature of Mathematical Morphology is the Fuzzy Mathematical Morphology (FMM), which is supported by concepts of fuzzy logic and fuzzy set theory [4-11]. Unlike bimodal logic, vagueness and uncertainty are qualities of this approach. Particularly, in predicate fuzzy logic, a truth value between 0 and 1 can be associated with each predicate, extending the boolean concept that the predicates should be only true or false.

Compensatory fuzzy logic (CFL) is a multivariate model, a particular case of the FMM, based on the replacement of t-norms and s-norms by conjunctions and disjunctions [12-13]. This is possible by relaxing con- strains imposed by t-norms and s-norms, which are by themselves also conjunctions and disjunctions.

By replacing t-norm and s-norm by conjunction and disjunction, respectively, we obtain the dilation and erosion operators for the Compensatory Fuzzy Mathematical Morphology (CMM), called compensatory dilation and compensatory erosion, respectively. Two different implementations of the CMM were presented at this moment $[12,21]$.

In this work new operators based on the definition of the CMM are presented, but replacing the supreme and infimum by logical operators, which allow for a linguistic interpretation of their meaning. We call the New Compensatory Morphological Operators. These operators are compared to the existing ones (MM and CMM) on their robustness against noise.

\section{Theorical Concepts}

The theory of Mathematical Morphology (MM) is a powerful tool in the Digital Image Processing field. A key aspect of this theory is the use of the structuring element (SE), a probe set that is used to test the image in several ways, generating information about its geometry. One of the reasons of the success of Mathematical Morphology is its simplicity of implementation: most of the operators can be built by combination of basic operators of negation, complement, dilation and erosion. The last two are considered the pillars of MM, and most of its variants are based on variants of these operators, as for example in the gray level morphology [14].

\subsection{Binary Mathematical Morphology}

Here we define the basic operators of the MM for binary images. A binary $X$ is a subset of $\square^{2}$, represented by its characteristic function $\chi_{X}: \square^{2} \rightarrow\{0,1\}$.

Let $A$ and $B$ two subsets of $U \subseteq \square^{2}$, the binary dilation of $A$ by the structuring element (SE) $B$, written as $D(A, B)$, is the set of points $x \in U$ such that $B_{x}$ has non empty intersection with $A[2]$ :

$$
D(A, B)=\left\{x \in U / B_{x} \cap A \neq \varnothing\right\}
$$


where $B_{x}=\{b+x / b \in B\}$ is called the traslation of $B$.

Dilating an image $A$ by the structuring element $B$ consists on the removal, from the background, of all the points $x$ for which $B_{x}$ is not included in A. It is equivalent to define the dilated image as the set of points $x$ such that $B_{x}$ intersects the image A.

Let $\mathrm{A}$ and $\mathrm{B}$ be two subsets of $U \subseteq \square^{2}$. The binary erosion of $A$ by the structuring element $B$, written as $E(A, B)$, is the set of points $x \in U$ such that $B_{x}$ is included in $A$ [2]:

$$
E(A, B)=\left\{x \in U / B_{x} \subseteq A\right\}
$$

To erode the image $A$ by the structuring element $B$ consists on reducing the set $A$ via a process of removal of points.

\subsection{Fuzzy Mathematical Morphology}

Several methodologies have been developed to extend binary MM to grayscale images. One of these extensions, based on fuzzy set theory, is the Fuzzy Mathematical Morphology (FMM) [4]. The FMM has proven to be a solid theory and has been applied successfully in biomedical image segmentation [15-18].

Operations between fuzzy sets are defined based on the conjunction and disjunction operators, applied to the membership values of these sets [19]. The values of the membership functions are numbers in the interval $[0,1]$. In most cases the images in gray levels are defined so that the gray level intensity at each pixel is an integer value belonging to the natural range 0,255 . Therefore, to be able to apply the FMM operators, gray level images must be modeled as fuzzy sets, with a change of scale to the range $[0,1]$. This process of scaling is called "fuzzification", while the reverse process is called "defuzzification". Usually the fuzzification function used, $g:\{0,1,2, \ldots, 255\} \rightarrow[0,1]$, is defined by:

$$
g(x)=\frac{x}{255}
$$

The reverse process by which the intensities of the gray levels of an image, belonging to the interval $[0,1]$, are brought back to the set $\{0,1,2, \ldots, 255\}$ is defined by the function $h:[0,1] \rightarrow\{0,1,2, \ldots, 255\}$ defined by:

$$
h(x)=[255 x]
$$

where $[[]: \square \rightarrow \square$ represents the integer part function, defined by:

$$
[a]=\sup \{k \in \square / k \leq a\}
$$

In the following sections $\mu$ and $v$ will denote two fuzzy sets, with membership functions $\mu: U \subseteq \square^{2} \rightarrow[0,1]$ and $v: U \subseteq \square^{2} \rightarrow[0,1]$, where the first one corresponds to a grayscale image and the second one determines the structuring element. Membership functions are obtained by applying fuzzification function over the gray scale images.

The literature that studies the extension of basic operators in binary images to gray levels image using the fuzzy set theory presents several approaches. Di Gesú, De Baets, Kerre, Bloch, Maître y Nachtegael are some of the authors which have developed several theories and have defined different formulas of the basic operators of the FMM [4-8,10,11,15]. Bloch and Maître have achieved the unification of all the models proposed by the authors mentioned previously, by the use of t-norms and s-norms. The following are the definitions of the basic operators of the FMM given by these authors.

Fuzzy dilation of the image $\mu$ by the SE $v$ [4]:

$$
\delta(\mu, v)(x)=\sup _{y \in U}[t(\mu(y), v(y-x))]
$$

where $t(a, b)$ is a t-norm [19].

Fuzzy erosion of the image $\mu$ by the EE $v$ [4]:

$\varepsilon(\mu, v)(x)=\inf _{y \in U}[s(\mu(y), c(v(y-x)))]$

where $s(a, b)$ is a s-norm and $c(a)$ is the fuzzy complement operator [20].

\subsection{Compensatory Fuzzy Mathematical Morphology}

Compensatory Fuzzy Mathematical Morphology (CMM) is a particular case of the FMM, based on the replacement of t-norms and s-norms by conjunctions and disjunctions of compensatory fuzzy logic (CFL) [13]. This is possible by relaxing the constraints imposed by t-norms and snorms, which are by themselves also conjunctions and disjunctions.

By replacing t-norm and s-norm by conjunction and disjunction, respectively, we obtain the dilation and erosion operators for the CMM:

$$
\begin{aligned}
& \delta(\mu, v)(x)=\sup _{y \in U}[C(\mu(y), v(y-x))] \\
& \varepsilon(\mu, v)(x)=\inf _{y \in U}[D(\mu(y), c(v(y-x)))]
\end{aligned}
$$

These operators are called compensatory dilation and compensatory erosion, respectively.

\subsection{New Operators of the CMM}

In this work we propose a new linguistic representation of CMM dilation and erosion operators, in a way that they can be associated to colloquial language, offering the following advantages:

- A colloquial expression for the operators, which gives clearer understanding of the effects of transformations, and the operations carried out in the process.

- A new paradigm for the morphological operators.

- A broader range of functions for the logical connectives, providing more implementation alternatives.

The proposal consists on replacing the definitions of the dilation and erosion operators, replacing the supremum 
and infimum by logic operations that model linguistically their meaning. Specifically, supremum is replaced by the "existential" quantifier and infimum is replaced by the "for all" quantifier. The whole process is described in the next section.

\subsubsection{New Dilation Operator}

Binary morphological dilation is defined by equation (1):

$$
D(A, B)=\left\{x \in U / B_{x} \cap A \neq \varnothing\right\}
$$

In this equation, the non-empty intersection between $\mathrm{B}$ and $\mathrm{A}, B_{x} \cap A \neq \varnothing$, can be expressed linguistically as "the intensity of a pixel in the output image is high when, after placing the SE over the original image, centered on such pixel, at least in one pixel of the region covered by the $S E$, the intensity of such pixel is high for both the original image and the translated SE.".

Let $\mu$ and $v$ functions valued between 0 and 1 , which indicate the truth value that a pixel has a high intensity, in the image and the SE respectively. They can be expressed with the following predicates: $\mu(y)=$ "the intensity is high in pixel y of the image to be processed" and $v(y)=$ "the intensity is high in the pixel y of the SE". If the image is a gray level image between 0 and 1, the relationship between the gray level and the predicate "the intensity is high in the pixel" is straightforward. For example, if the pixel has intensity 0.45 , the truth value for the predicate would be also 0.45 .

Let the translation $v_{x}$ of the fuzzy set $v$ by the element $x \in \square^{n}$ be given by:

$$
v_{x}(y)=v(y-x) \quad \forall y \in \square^{n}
$$

From the previous definition, the linguistic expression can be written in the following ways:

$$
\begin{gathered}
\exists y \in U: v_{x}(y) \wedge \mu(y) \\
\exists y \in U: \mu(y) \wedge v(y-x) \\
\exists y \in U: C(\mu(y), v(y-x))
\end{gathered}
$$

As a consequence, the new dilation operator is defined as:

$$
\delta(\mu, v)(x)=\underset{y \in U}{\exists} y:\{C(\mu(y), v(y-x))\}
$$

\subsubsection{New Erosion Operator}

In the same way we can develop the binary erosion, as defined in equation (2), resulting in:

$$
E(A, B)=\left\{x \in U / B_{x} \subseteq A\right\}
$$

The inclusion operator $B_{x} \subseteq A$, can be expressed linguistically in the following way: "the intensity of a pixel in the output image is high when, after placing the SE over the original image, centered on such pixel, for all the pixels of the region covered by the SE, if the intensity of such pixels is high for the translated SE, the it should be high for the original image."
Considering the functions $\mu$ and $v_{x}$ described previously, this last linguistic expression can be written as:

$$
\begin{gathered}
\forall y \in U: v_{x}(y) \Rightarrow \mu(y) \\
\forall y \in U: v(y-x) \Rightarrow \mu(y) \\
\forall y \in U: c[v(y-x)] \vee \mu(y) \\
\forall y \in U: D(\mu(y), c(v(y-x)))
\end{gathered}
$$

As a consequence, the new erosion operator is defined as:

$$
\varepsilon(\mu, v)(x)=\underset{y \in U}{\forall} y:\{D(\mu(y), c(v(y-x)))\}
$$

\subsubsection{Quantifiers}

Equations 11 and 12 present the existential and universal operators. Such operators are defined from the disjunction and conjunction in the following way [21]:

$$
\begin{aligned}
& \underset{x \in U}{\exists} p(x)=D\{p(x): x \in U\} \\
& \underset{x \in U}{\forall} p(x)=C\{p(x): x \in U\}
\end{aligned}
$$

Several implementations of these quantifiers can be obtained by using different conjunctions and disjunctions. In this work we use operators from Compensatory Logic, obtaining new morphological operators, not previously defined in literature.

It is important to note that, in the case of dilation, where the existential quantifier is defined by a disjunction, if such disjunction were the maximum, then this new definition is equivalent to the model proposed by Bloch and Maître [4]. The case is similar for erosion if the conjunction is the minimum operator.

Because of the previous analysis, this new definition generalizes the previous definition of fuzzy operators proposed by Bloch and Maître [4].

\section{Experimental Design}

This section presents the experimental results of the robustness comparisons. The operators compared are erosion and dilation, for the new definition against the classical ones: mathematical morphology and compensatory morphology. There are no performance evaluation, like in noise removal or other tasks, but an analysis of how much noise in the original images affects the results of the operators, compared to not noisy ones.

We say that an operator is robust against noise if the result of applying it to a noisy image is similar to the result of applying it to the original image. The degree of similarity determines the robustness. The closer are the results from the noisy image to the results from the noiseless image, the more robust is the operator against noise. In this work, the operators of the different approaches (MM, CMM, new CMM) are compared with respect to their robustness. 
For the analysis we used magnetic resonance imaging (MRI). Medical imaging has become an important application field of image processing. These kind of images, and in particular MRI have two remarkable features: they include noise and distortion inherent to the acquisition equipment and they present high variability between equipment settings. Considering these features, fuzzy operators have been proved being more robust than other approaches. Because of these properties, we selected the MRI for this study.

Mechanisms of generation of this kind of images, MR, TAC, etc, produce images with high level of imprecision in the boundaries between the tissues and structures.

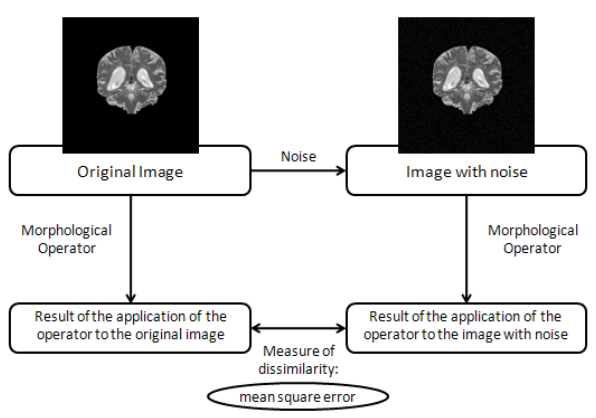

Fig. 1: Scheme of the experiments design.

In each experiment we computed the difference between the results of the operator applied on the original (noiseless) image and the noisy image. Operators applied were erosion and dilation. Implementations under comparison were classic MM, CMM (using both geometric and algebraic mean) and the new CMM (also using both geometric and algebraic mean).

We compared results for 7 levels of noise, over 100 replicates to average the results on random application of noise. Error measure used was the mean square error (MSE) on the pixel values (between results on noisy and noiseless images). This process was repeated on 10 different images.

The following list provides a detailed description of the experimental setup:

- Images: we used 10 magnetic resonance images, acquired with a Tesla 1.5 equipment The protocol included coronal and axial images, weighted in T2 (TR/TE!/TE = 3,500/32/96 ms). As explained before, we used this kind of images because of the noisy nature of them. Figure 2 shows some examples of the images used for the analysis.

- Conjunction and disjunction operators: Operations were implemented presented in tables 1 and 2 [12,21].

- Noise: we use independent Gaussian noise with distribution $N\left(0, \sigma^{2}\right)$. The 6 values of variance used were: $\sigma^{2}=50, \quad \sigma^{2}=100, \quad \sigma^{2}=150, \quad \sigma^{2}=200, \quad \sigma^{2}=250 \quad \mathrm{y}$ $\sigma^{2}=300$.

- Iterations: the analysis was repeated 100 times to average over random generation of random noise.

In first place we studied the dilation operator. We first dilated the original noiseless images with the different implementations. Then we added Gaussian noise and applied again the dilation operator. Finally we computed the MSE on the two results.

For dilation the processing was similar, using the disjunctions of tables 1 and 2 .

To analyze the results under different conditions, we changed the dimension of the SE. We designed five experiments, including five SE. For the MM flat structuring elements (SE) of size $3 \times 3,5 \times 5,7 \times 7,11 \times 11$ and $15 \times 15$ were used. For the FMM symmetric SE of the same size were used. They were the fuzzification of the flat SE of the MM, using a Gaussian function to give them the bell shape, as seen in figure 3. Such SE should help to discriminate some structures present in the original image.

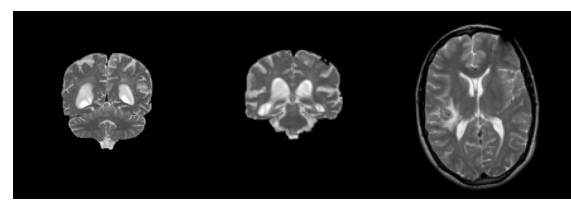

Fig. 2: Examples of brain MRI used for the analysis of basic morphological operators.

Table 1: Conjunction and disjunction of Geometric Mean Based Compensatory Fuzzy Logic (GMBCFL).

\begin{tabular}{|c|c|}
\hline & CFL (Geometric Mean) \\
\hline Conjunction & $\left(x_{1} \cdot x_{2} \ldots \ldots x_{n}\right)^{\frac{1}{n}}$ \\
$C\left(x_{1}, x_{2}, \ldots, x_{n}\right)$ & $1-\left[\left(1-x_{1}\right) \cdot\left(1-x_{2}\right) \ldots . .\left(1-x_{n}\right)\right]^{\frac{1}{n}}$ \\
\hline Disjunction \\
$D\left(x_{1}, x_{2}, \ldots, x_{n}\right)$ & \\
\hline
\end{tabular}

Table 2: Conjunction and disjunction of Arithmetic Mean Based Compensatory Logic (AMBCFL).

\begin{tabular}{|c|c|}
\hline $\begin{array}{c}\text { Conjunction } \\
C\left(x_{1}, x_{2}, \ldots, x_{n}\right)\end{array}$ & CFL (Arithmetic Mean) \\
\hline $\begin{array}{c}\text { Disjunction } \\
D\left(x_{1}, x_{2}, \ldots, x_{n}\right)\end{array}$ & $1-\left[\min \left(x_{1}, x_{2}, \ldots, x_{n}\right) \cdot \frac{\sum_{i=1}^{n} x_{i}}{n}\right]^{\frac{1}{2}}$ \\
\hline
\end{tabular}

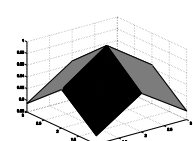

(a)

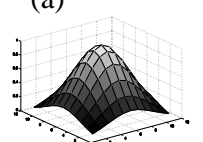

(d)

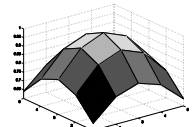

(b)

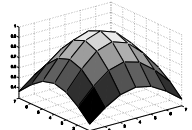

(c)
Fig. 3: 3D view of the 5 structuring elements used for the FMM. (a) $3 \times 3$. (b) $5 \times 5$. (c) $7 \times 7$. (d) $11 \times 11$. (e) $15 \times 15$.

\section{Results}

This section shows the results from performance analysis. As an example, figure 4 shows a MR image and the same image with random noise added (variance $=300$ ). Figure 
5 shows some image results of the dilation with a $7 \times 7$ SE. The left column shows the dilation of the original noiseless image, for the different implementations of MM, while the right column shows the same results over the noisy image. Figure 7 shows a similar example for the erosion operator, with same level of noise and implementations.

Tables 3 to 6 show the highest and lowest error, first for dilation and then for erosion, as a function of the amount of noise (variance) and the implementation: $\mathrm{C} 1$ and $\mathrm{C} 2$ for the $\mathrm{CMM}, \mathrm{NC} 1$ and $\mathrm{NC} 2$ for the new operators, and MM for classic MM. These matrices were computed in the following way:

- For each image, variance and operator, the arithmetic mean of the errors was computed from the 100 iterations.

- For each variance and operator, the arithmetic mean of the errors was computed over all images.

- The table displays the average errors, with noise variance in the columns and operators in the rows.

Results are displayed graphically in figures 6 and 8 . Vertical segments over the error lines indicate the errors average and dispersion.

Figure 9 shows graphically the box-plot of error rates comparing the classic mathematical morphology (MM) and the new compensatory morphology (New CMM) based on the GMBCFL, for both dilation and erosion, and for the largest amount of noise $\left(\sigma^{2}=300\right)$, across all the structuring element sizes. We choose this last operator because they have the minimum error. Here we can see a significant decrease on error rates, or differences between the original and noisy images after application of the morphological operators.

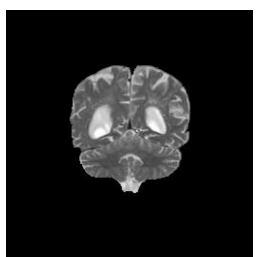

(a)

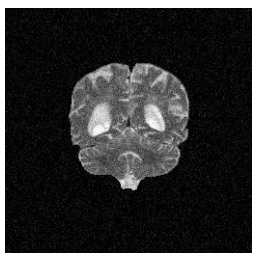

(b)
Fig. 4: Images that are used to display the results. (a) Original Image. (b) Image with noise using a variance equal to 300.

Tabla 3: Lowest error for dilation. The name of the operators that reached the minimum is denoted between parenthesis next to the error.

\begin{tabular}{|c|c|c|c|}
\hline SE & \multicolumn{3}{|c|}{$\sigma^{2}$} \\
\hline & 50 & 100 & 150 \\
\hline $3 \times 3$ & (NC1) 0.00034 & (NC1) 0.00064 & (NC1) 0.00093 \\
\hline $5 \times 5$ & (NC1) 0.00033 & (NC1) 0.0005 & (NC1) 0.00066 \\
\hline $7 \times 7$ & (NC1) 0.00068 & (NC1) 0.0009 & (NC1) 0.0011 \\
\hline $11 \times 11$ & (NC2) 0.00312 & (NC2) 0.00281 & (NC1) 0.00331 \\
\hline $15 \times 15$ & (NC2) 0.00157 & (NC2) 0.00334 & (NC2) 0.00529 \\
\hline \multirow[t]{2}{*}{ SE } & \multicolumn{3}{|c|}{$\sigma^{2}$} \\
\hline & 200 & 250 & 300 \\
\hline $3 \times 3$ & (NC1) 0.00122 & (NC1) 0.00152 & (NC1) 0.00182 \\
\hline $5 \times 5$ & (NC1) 0.00081 & (NC1) 0.00097 & (NC1) 0.00113 \\
\hline $7 \times 7$ & (NC1) 0.00128 & (NC1) 0.00146 & (NC1) 0.00166 \\
\hline
\end{tabular}

\begin{tabular}{|c|c|c|c|}
\hline $11 \times 11$ & $(\mathrm{NC} 1) 0.00373$ & $(\mathrm{NC} 1) 0.00412$ & $(\mathrm{NC} 1) 0.00452$ \\
\hline $15 \times 15$ & $(\mathrm{NC} 1) 0.00733$ & $(\mathrm{NC} 1) 0.00802$ & $(\mathrm{NC} 1) 0.0087$ \\
\hline
\end{tabular}

Tabla 4: Highest error for dilation. The name of the operators that reached the maximum is denoted between parenthesis next to the error. No name is indicated when the maximum was reached by MM.

\begin{tabular}{|c|c|c|c|}
\hline SE & \multicolumn{3}{|c|}{$\sigma^{2}$} \\
\hline & 50 & 100 & 150 \\
\hline $3 \times 3$ & 0.00382 & 0.00825 & 0.01305 \\
\hline $5 \times 5$ & 0.00396 & 0.00884 & 0.01439 \\
\hline $7 \times 7$ & 0.00388 & 0.00876 & 0.01438 \\
\hline $11 \times 11$ & 0.00364 & 0.00828 & 0.01359 \\
\hline $15 \times 15$ & 0.00477 & 0.00795 & 0.01321 \\
\hline \multicolumn{4}{|c|}{} \\
\hline SE & 200 & $\sigma^{2}$ \\
\hline \multicolumn{4}{|l|}{250} \\
\hline $3 \times 3$ & 0.01810 & 0.02344 & 0.02888 \\
\hline $5 \times 5$ & 0.02035 & 0.02660 & 0.03319 \\
\hline $7 \times 7$ & 0.02042 & 0.02697 & 0.03376 \\
\hline $11 \times 11$ & 0.01958 & 0.02600 & 0.03272 \\
\hline $15 \times 15$ & (NC1) 0.01899 & 0.02518 & 0.03153 \\
\hline
\end{tabular}

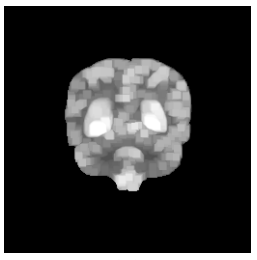

(a)

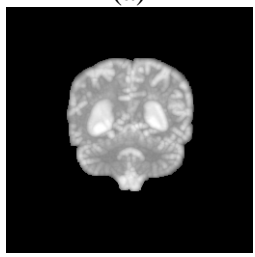

(c)

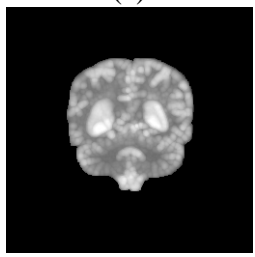

(e)

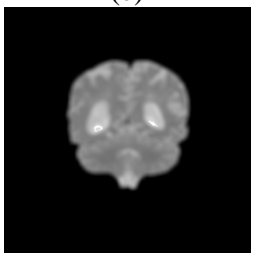

(g)

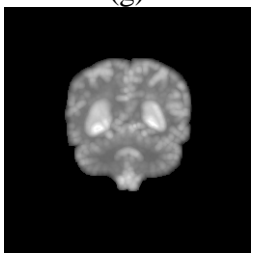

(i)

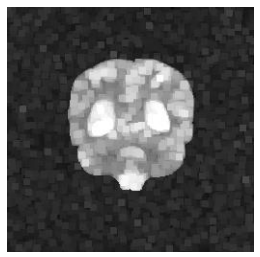

(b)

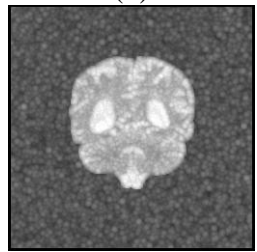

(d)

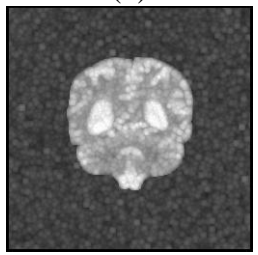

(f)

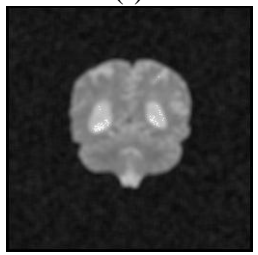

(h)

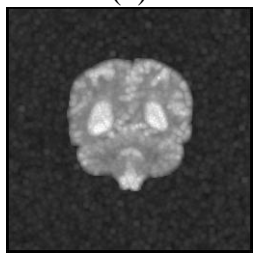

(j)
Fig. 5: Visualization of the dilation results using a SE of size $7 \times 7$ and variance equal to 300 to generate the noise. (a)-(b) 
Morphological dilation. (c)-(d) Compensatory dilation using the conjunction of the GMBCFL. (e)-(f) Compensatory dilation using the conjunction of the AMBCFL. (g)-(h) New compensatory dilation using the conjunction of the GMBCFL. (i)-(j) New compensatory dilation using the conjunction of the AMBCFL.

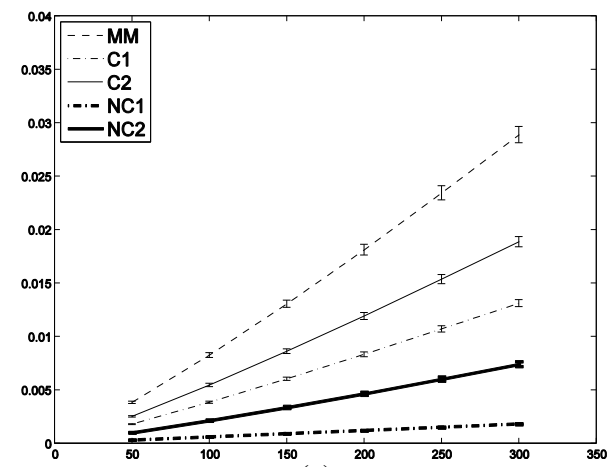

(a)

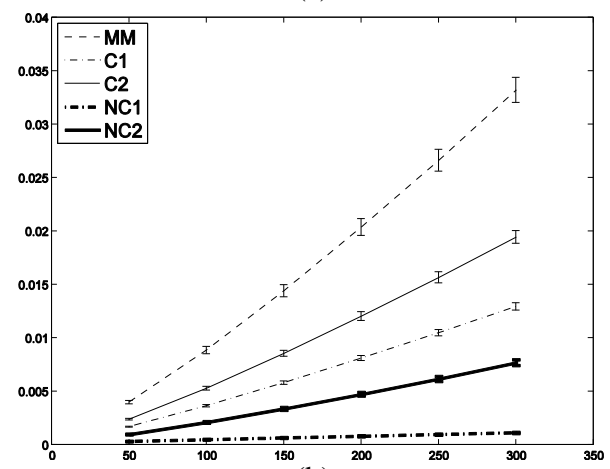

(b)

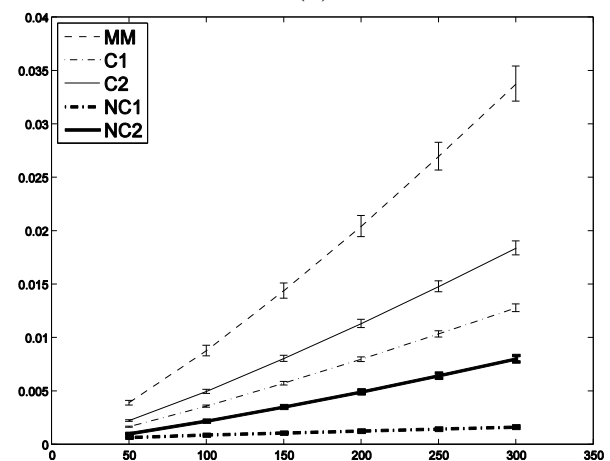

(c)

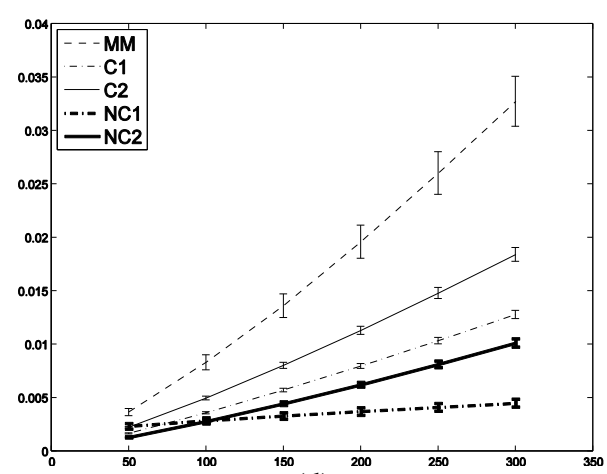

(d)

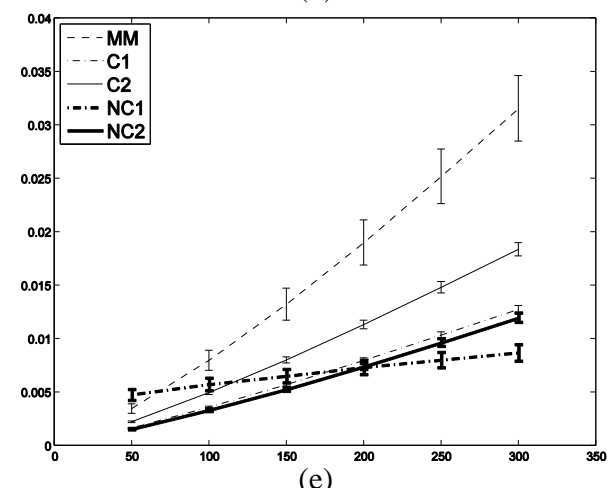

Figure 6: Error graphs for dilation. a) $3 \times 3 \mathrm{SE}$; b) $5 \times 5 \mathrm{SE}$; c) 7x7 $\mathrm{SE}$; d) $11 \times 11 \mathrm{SE}$; e) $15 \times 15 \mathrm{SE}$

Table 5: Lowest error for erosion. The name of the operators that reached the minimum is denoted between parenthesis next to the error.

\begin{tabular}{|c|c|c|c|}
\hline SE & \multicolumn{3}{|c|}{$\sigma^{2}$} \\
\hline & 50 & 100 & 150 \\
\hline $3 \times 3$ & (NC1) 0.00030 & (NC1) 0.00057 & (NC1) 0.00083 \\
\hline $5 \times 5$ & (NC1) 0.00012 & (NC1) 0.00024 & (NC1) 0.00035 \\
\hline $7 \times 7$ & (NC1) 0.00010 & (NC1) 0.00021 & (NC1) 0.00031 \\
\hline $11 \times 11$ & (NC1) 0.00018 & (NC1) 0.00042 & (NC1) 0.00063 \\
\hline $15 \times 15$ & (NC1) 0.00032 & (NC1) 0.00079 & (NC1) 0.00120 \\
\hline \multirow[t]{2}{*}{ SE } & \multicolumn{3}{|c|}{$\sigma^{2}$} \\
\hline & 200 & 250 & 300 \\
\hline $3 \times 3$ & (NC1) 0.00109 & (NC1) 0.00135 & (NC1) 0.00162 \\
\hline $5 \times 5$ & (NC1) 0.00046 & (NC1) 0.00057 & (NC1) 0.00068 \\
\hline $7 \times 7$ & (NC1) 0.00041 & (NC1) 0.00050 & (NC1) 0.00060 \\
\hline $11 \times 11$ & (NC1) 0.00082 & (NC1) 0.00099 & (NC1) 0.00115 \\
\hline $15 \times 15$ & (NC1) 0.00153 & (NC1) 0.00184 & (NCl) 0.00215 \\
\hline
\end{tabular}

Tabla 6: Highest error for erosion. The name of the operators that reached the maximum is denoted between parenthesis next to the error. No name is indicated when the maximum was reached by MM.

\begin{tabular}{|c|c|c|c|}
\hline SE & \multicolumn{3}{|c|}{$\sigma^{2}$} \\
\hline & 50 & 100 & 150 \\
\hline $3 \times 3$ & 0.00396 & 0.00872 & 0.01388 \\
\hline $5 \times 5$ & 0.00467 & 0.01092 & 0.01796 \\
\hline $7 \times 7$ & 0.00512 & 0.01234 & 0.02061 \\
\hline $11 \times 11$ & 0.00546 & 0.01357 & 0.0228 \\
\hline $15 \times 15$ & 0.00534 & 0.01363 & 0.02302 \\
\hline \multicolumn{5}{|c|}{} \\
\hline SE & 200 & $\sigma^{2}$ & \\
\hline \multicolumn{5}{|c|}{} & 250 & 300 \\
\hline $3 \times 3$ & 0.01933 & 0.02498 & 0.03085 \\
\hline $5 \times 5$ & 0.02547 & 0.03336 & 0.04156 \\
\hline $7 \times 7$ & 0.02942 & 0.03880 & 0.0485 \\
\hline $11 \times 11$ & 0.03302 & 0.04383 & 0.05516 \\
\hline $15 \times 15$ & 0.03348 & 0.04459 & 0.05604 \\
\hline
\end{tabular}



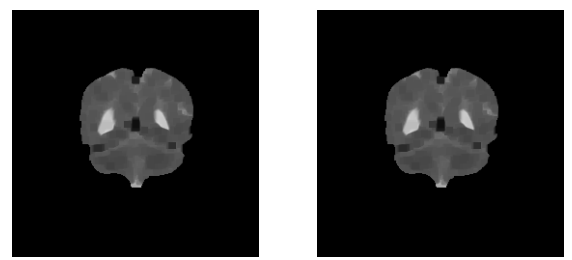

(a)

(b)
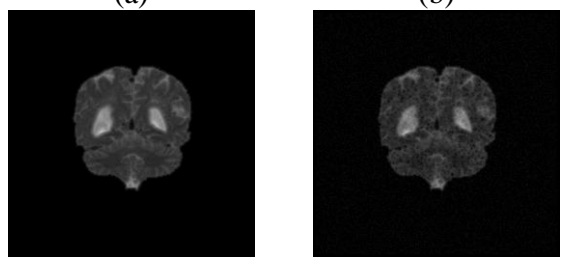

(c)

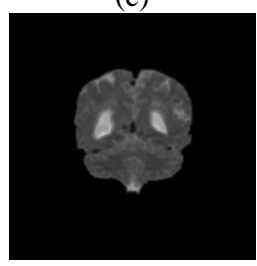

(e)

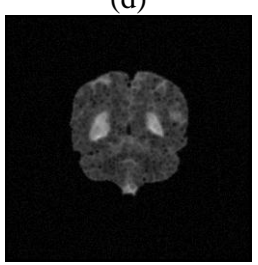

(f)

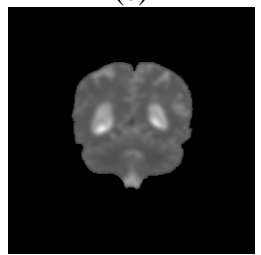

$(\mathrm{g})$

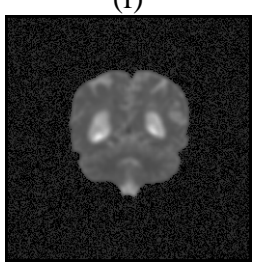

(h)

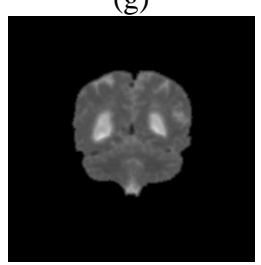

(i)

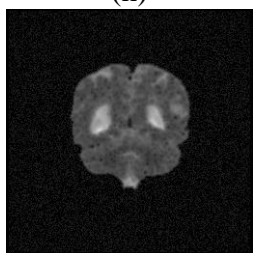

(j)

Fig. 7: Visualization of the erosion results using a SE of size $7 \times 7$ and variance equal to 300 to generate the noise. (a)-(b) Morphological erosion. (c)-(d) Compensatory erosion using the conjunction of the GMBCFL. (e)-(f) Compensatory erosion using the conjunction of the AMBCFL. (g)-(h) New compensatory erosion using the conjunction of the GMBCFL. (i)-(j) New compensatory erosion using the conjunction of the AMBCFL.

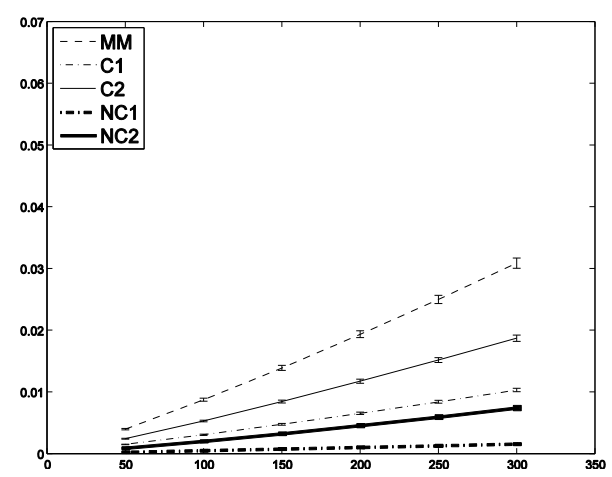

(a)

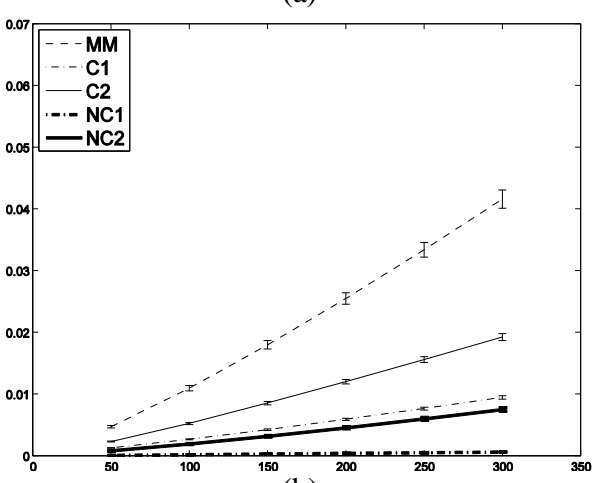

(b)

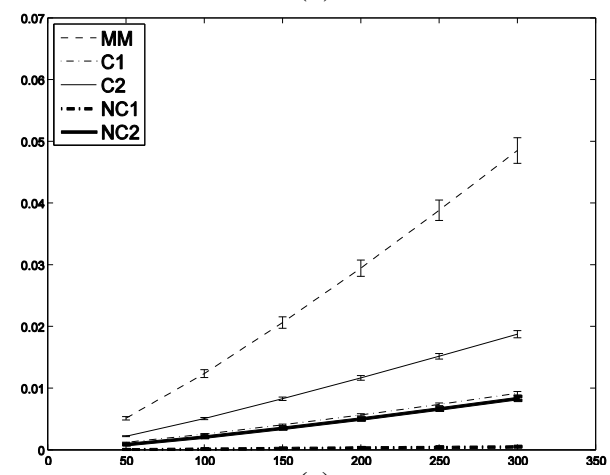

(c)

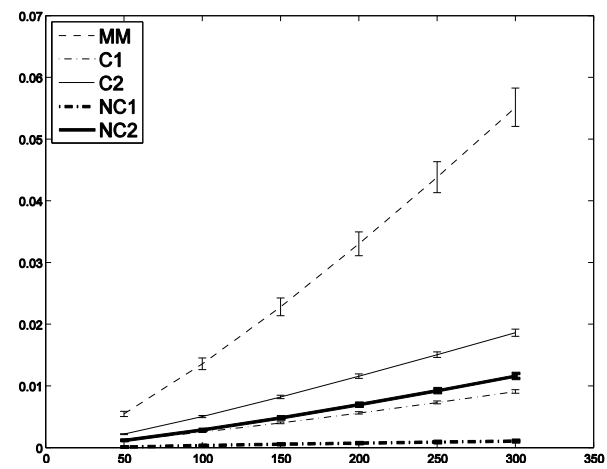

(d)

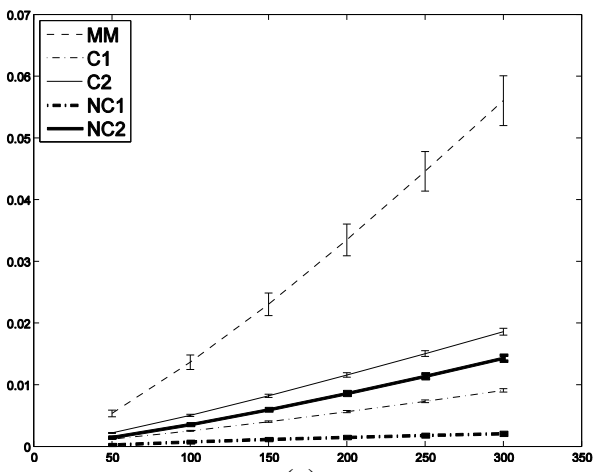

(e)

Figure 8: Graphs with errors for erosion. a) $3 \times 3 \mathrm{SE}$; b) $5 \times 5 \mathrm{SE}$; c) $7 \times 7 \mathrm{SE}$; d) $11 \times 11 \mathrm{SE}$; e) $15 \times 15 \mathrm{SE}$ 


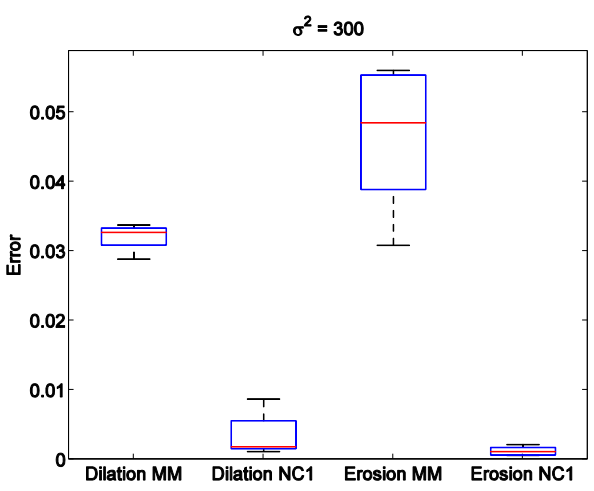

Fig. 9: Error rates box-plot comparing the classic mathematical morphology (MM) and the new compensatory mathematical morphology (New CMM) using LDCMG, for both dilation and erosion, and for the largest amount of noise $\left(\sigma^{2}=300\right)$, across all the structuring element sizes.

\section{Discussion}

From the results obtained in the experimental section, developed to analyze the robustness of the dilation operators, we can observe that in most cases MM implementation displays larger error than CMM and the new CMM implementation, with this contrast increasing with increasing variance (used to generate the noise). This means that results of the dilation operator, when applied to noisy images, are closer to the result on noiseless image for the CMM and new CMM implementations, than for the classic MM (this happens in the $96.67 \%$ of the cases). In the other $3.33 \%$ of the cases the worst performance was obtained by the new CMM based on geometric mean.

Implementations with lowest error, for dilation, are the new CMM with both conjunctions. 83\% corresponds to LDCMG and 17\% corresponds to LDCMA.

For erosion, classic MM has the worst performance for all cases. The best operator is the new CMM based on LDCMG.

It is important to note that operators defined in the new CMM, using the LDCMA disjunction, show always lowest error than classic MM.

In the graphs we can also observe that average error and its dispersion increase for increasing variance in noise generation.

From the previous discussion, we can conclude that for the two operators under analysis, erosion and dilation, the new CMM shows more robustness against noise than the $\mathrm{MM}$ and CMM implementations, on the images and noise models used for this study.

Therefore, the presence of noise in the images should have less effect on compensatory operators than in classic and Fuzzy ones.

\section{Conclusions}

In this work we defined new compensatory morphological operators of erosion and dilation, based on the substitution of the supreme and infimum by the existential and universal quantifiers, which added a linguistic representation for such operators.

The results obtained on robustness, for erosion and dilation, relative to additive Gaussian noise, shows an important improvement relative to classic operators. This evidence supports the importance of using fuzzy operators for situations with noise and incertitude.

Future work will include the analysis of performance of this paradigm relative to other operators, images and noise.

\section{References}

[1] R. González, R. Woods, Digital Image Processing, Addison Wesley, 1996.

[2] J. Serra, Image analysis and mathematical morphology, vol. 1, Academic Press, London, 1982.

[3] J. Serra, Image analysis and mathematical morphology, vol. 2, Academic Press, London, 1988.

[4] I. Bloch, H. Maitre, "Fuzzy mathematical morphologies: A comparative study", Pattern Recognition, vol. 28, pp. 1341-1387, 1995.

[5] I. Bloch, "Duality vs Adjunction and General Form for Fuzzy Mathematical Morphology", in: S.B. Heidelberg, (Ed.), Fuzzy Logic and Applications, pp. 354 $-361,2006$.

[6] I. Bloch, "Duality vs. adjunction for fuzzy mathematical morphology and general form of fuzzy erosions and dilations", Fuzzy Sets and Systems, vol. 160, pp. 1858-1867, 2009.

[7] B. De Baets, E. Kerre, M. Gupta, "The Fundamentals of Fuzzy Mathematical Morphology. Part 1: Basic Concepts", International Journal of General Systems, vol. 23, pp. 155 - 171, 1995.

[8] B. De Baets, E. Kerre, M. Gupta, "The Fundamentals of Fuzzy Mathematical Morphology. Part 2: Idempotence, Convexity and Decomposition", International Journal of General Systems, vol. 23, pp. 307 $-322,1995$

[9] B. De Baets, "Idempotent closing and opening operations in fuzzy mathematical morphology", Proceedings of ISUMA - NAFIPS '95 The Third International Symposium on Uncertainty Modeling and Analysis and Annual Conference of the North American Fuzzy Information Processing Society, pp. 228-233, 1995.

[10] V. Di Gesu, "Mathematical morphology and image analysis: a fuzzy approach", Proceedings of the Workshop pn Knowledge-Based Systems and Models og Logical Reasoning, Cairo, Egypt, 1988.

[11] V. Di Gesu, M.C. Maccarone, M. Tripiciano, "Mathematical Morphology based on Fuzzy Operators", in: R. Lowen, M. Roubens, (Eds.), Fuzzy Logic, Kluwer Academic Publishers, pp. 477-486, 1993.

[12] A. Bouchet, J.I. Pastore, R.E. Andrade, M. Brun, V. Ballarin, "Arithmetic Mean Based Compensatory Fuzzy Logic", International Journal of Computation- 
al Intelligence and Applications (IJCIA), vol. 10, pp. 231-243, 2011.

[13] A. Bouchet, J.I. Pastore, R.E. Andrade, M. Brun, V. Ballarin, "Compensatory Logic applied to Digital Image Processing", in: R.E. Andrade, J.M. Gómez, A.R. Valdéz, (Eds.), Towards a trans-disciplinary technology of Business and Organizational Intelligence: Gathering Knowledge Discovery, Knowledge Management and Decision, Shaker Verlag, Aachen, University of Oldenburg, Alemania, pp. 226-239, 2011.

[14] J.A. Bangham, S. Marshall, "Image and Signal processing with mathematical morphology", IEE Electronics \& Communication Engineering Journal, vol. 10, pp. 117-128, 1998.

[15] E.E. Kerre, M. Nachtegael, Fuzzy Techniques in Image Processing, New York, 2000.

[16] E. Blotta, A. Bouchet, M. Brun, V. Ballarin, "Characterization of bio-dynamic speckles through classical and fuzzy mathematical morphology tools", Signal Processing, vol. 93, pp. 1864-1870, 2013.

[17] A. Bouchet, J. Pastore, V. Ballarin, "Segmentation of Medical Images using Fuzzy Mathematical
Morphology", Journal of Computer Science and Technology, vol. 7, pp. 256-262, 2007.

[18] J.P. Gasparri, A. Bouchet, G. Abras, V. Ballarin, J.I. Pastore, "Medical Image Segmentation using the HSI color space and Fuzzy Mathematical Morphology”, Journal of Physics: Conference Series, vol. 332, pp. 1-9, 2011.

[19] D. Dubois, H. Prade, Fuzzy Sets and Systems: Theory and Applications, Academic Press Inc New York, 1980.

[20] E.P. Klement, R. Mesiar, E. Pap., "Triangular norms. position paper I: basic analytical and algebraic properties", Fuzzy Sets and Systems, vol. 143, pp. 526, 2004.

[21] R. Espin Andrade, E. Fernández González, La Lógica Difusa Compensatoria: Una Plataforma para el Razonamiento y la Representación del Conocimiento en un Ambiente de Decisión Multicriterio, Análisis Multicriterio para la Toma de Decisiones: Métodos y Aplicaciones. Coedición: editorial Plaza y Valdes / Editorial Universidad de Occidente (2009). 